\title{
VASCULAR ANOMALIES IN NEWBORNS: CLINICAL PRESENTATION, COMPLICATIONS, AND PECULIARITIES OF THERAPY
}

DOl: $10.36740 /$ WLek202009207

\author{
Iryna M. Benzar' ${ }^{1}$, Anatolii F. Levytskyi' ${ }^{1}$, Daria S. Diehtiarova' ${ }^{1}$, Larysa Ya. Fedoniuk², Yaroslav S. Stravskyy², \\ Daria A. Merkulovaa ${ }^{3}$, Petro A. Sas ${ }^{2}$ \\ 'BOGOMOLETS NATIONAL MEDICAL UNIVERSITY, KYIV, UKRAINE \\ 2. HORBACHEVSKY TERNOPIL NATIONAL MEDICAL UNIVERSITY, TERNOPIL, UKRAINE \\ ${ }^{3}$ NATIONAL PIROGOV MEMORIAL MEDICAL UNIVERSITY, VINNYTSIA, UKRAINE
}

\begin{abstract}
The aim: To analyze cases of vascular anomalies in newborns retrospectively to study their main clinical signs and to determine indications for urgent treatment. Materials and methods: A retrospective review of 281 pediatric vascular anomalies diagnosed between 2011 and 2019 was performed. The results of clinical examination, prenatal history, laboratory, sonography, and radiological data were evaluated.

Results: Clinical manifestations in the newborn period documented in 170 (60.5\%) patients, anomalies were visualized prenatally in $8.9 \%$ cases. Five newborns with head and neck LMs required urgent treatment, which amounted $7.9 \%$ of all newborns with LMs. The indication for urgent surgery was acute respiratory failure. Partial malformation resection with tracheostomy was performed to those patients, along with intraoperative injection of sclerotherapeutic agents and argon coagulation of residual malformation tissues. EXIT procedure was performed in one case. No correlation $(p=0.2)$ was found between the number of skin lesions and the severity of liver lesions in children with multifocal hepatic hemangiomas, congestive cardiac failure suddenly occurs in one patient in the third week of life. Two newborns with diffuse hepatic hamangioma were manifested right after the birth with clinical signs of liver failure, hypothyreosis, and cardiac failure. Short courses of corticosteroids performed in 7 patients with KHE and Kasabach-Merritte fenomemn for thrombocytopenia correction.

Conclusions: Clinical signs of vascular anomalies in neonatal period were seen in $57.5 \%$ children with vascular anomalies, and only $2.9 \%$ of them required treatment. Treatment itself is advantageous only in cases when life-threatening complications develop, such as airways obstruction, cardiac and/or liver failure, thrombocytopenia.
\end{abstract}

KEY WORDS: vascular anomalies, vascular malformations, congenital and infantile hemangioma, newborns

\section{INTRODUCTION}

According to renewed International Society for the Study of Vascular Anomalies (ISSVA) 2018 classification, all vascular anomalies are divided into two basic groups: vascular malformations and vascular tumors [1].

Mentioned anomalies are fundamentally different not only by their anatomical, histological and pathophysiological features, but also by clinical course. This fact places a special emphasis on the importance of clear division of these two groups to choose the appropriate treatment tactics [2].

Dichotomous division to vascular tumors and vascular malformations was originally proposed in 1982 [3], and this basic principle is kept in modern classification. Along with that, knowledge of vascular anomalies has considerably improved through following decades $[4,5]$. Genetic risk loci for many of them were determined, new nosological units were described, numerous mechanisms pathogenesis were discovered, and new treatment methods were implemented. In earlier publications for instance it was suggested that the basic pathogenetic causes of lymphatic malformations (LMs) were disorders in embryological development of lymphatic vessels with following absence of lymphatic channels and venous vessels communications [6]. In recent publications arguments are provided for LMs are not the result of vasculogenesis defects but of sporadic genetic abnormalities in specific cells [7] that will create a possibility of target therapy.

Despite modern medical science achievements, there are issues of diagnostic and treatment tactics of vascular anomalies in newborns, as typical clinical presentation is not always seen, there is little data about ongoing course of disease and limitations for additional invasive methods of examination.

\section{THE AIM}

Aim of the study was to analyze clinical cases of vascular anomalies in newborns retrospectively to study their main clinical signs and to determine indications for urgent treatment.

\section{MATERIALS AND METHODS}

A retrospective study was conducted to review the medical documents of 281 pediatric patients with vascular anomalies. 


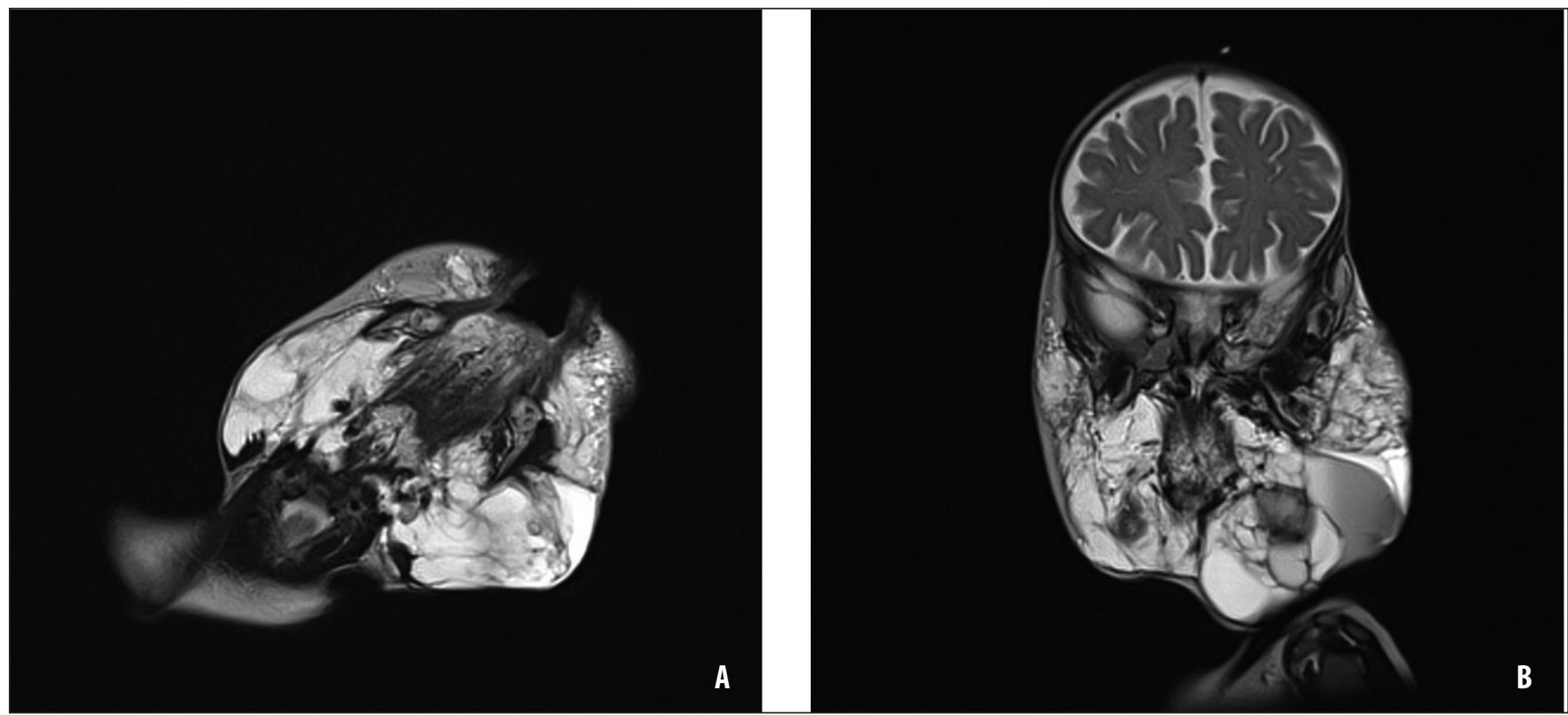

Fig. 1. Newborn male with head and neck LM. Axial (A) and coronal (B) T2-weighted MRI showing diffuse infiltrative affection of all tissues by macroand microcystic mass.

The diagnosis and treatment of all cases were performed in one hospital in a period from January 2011 to December 2019. The group of patients aged $0-28$ days (newborns) with symptoms of vascular anomalies was selected. The results of clinical examination, prenatal history, laboratory, sonography, computed tomography (CT) and magnetic resonance imaging (MRI) data were evaluated. Where possible, histological confirmation was included. Vascular malformations were diagnosed in 198 (70.5\%) and vascular tumors in 83 (29.5\%) children. Distribution of vascular malformations was as follows: LMs were diagnosed in 145 (73.2\%) children, venous malformations (VMs) - in 39 (19.7\%) and arteriovenous malformations (AVMs) in 14 (7.1\%) patients. Among 83 patients with vascular tumors infantile hemangioma (IH) was predominant, diagnosed in $68(81.9 \%)$ children, congenital hemangioma $(\mathrm{CH})$ - in $9(10.9 \%)$ and kaposiform hemangioendotheliomas (KHE) in $6(7.2 \%)$ patients. A detailed analysis of clinical cases was performed to determine those 170, where clinical presentation appeared at the age of 0-28 days. The results of fetal sonography were available in 180 patients, and there was one fetal MRI.

The statistical analysis was performed using IBM SPSS Statistics, version 23. The data is presented as an average of 95 percent confidence interval or standard deviation. The qualitative data were analyzed in a univariate analysis, with the Pearson's correlation coefficient. Independent predictors of morbidity in newborn patients were determined using multivariable logistic regression analysis. Statistical significance was defined as $\mathrm{p}<0.05$.

\section{RESULTS}

Clinical manifestations of vascular anomalies in the newborn period documented in 170 (60.5\%) patients, anomalies were visualized prenatally in $8.9 \%$ cases. Among
145 children with LMs clinical signs were detected in neonatal period in 78 (53.8\%) cases. 19 (24.4\%) of them were diagnosed prenatally. The most common were head and neck LMs - $63(80.8 \%)$ patients. Less frequently LMs were seen in axilla $(\mathrm{n}=8 ; 10,3 \%)$ either in pelvis and extremities $(n=7 ; 8.9 \%)$. The first clinical sign of cystic LMs was disfigurement owing to a mass in affected anatomical regions. Complications were registered in 17 (27.0\%) children with head and neck LMs, including in particular upper airways compression followed by acute respiratory failure $(n=5$, $6.4 \%)$, transitory stridor $(\mathrm{n}=8,12.7 \%)$, transitory dysphagia $(\mathrm{n}=2 ; 3.2 \%)$, intermittent lymphorrhea in children with affection of oral mucosa $(n=2,3.2 \%)$. Five newborns with head and neck LMs required urgent treatment, which amounted $6.4 \%$ of all newborns with LMs. MRI showed that mass is represented by cysts of different diameters and multiple septa, with average size of $301,05 \pm 56,28 \mathrm{sm}^{2}$, that affect head and neck tissues on both sides and spread beyond fascial leaves (Fig.1). The sizes of LMs in this group of patients were almost double the average of $153.0 \pm 114.2$ $\mathrm{sm}^{2}(\mathrm{p}<0.05)$.

The indication for urgent surgery was clinical presentation of acute respiratory failure, caused by disorders in respiration mechanics and external obstruction of upper airways. Partial malformation resection with tracheostomy was performed to those patients, along with intraoperative injection of sclerotherapeutic agents (OK-432, bleomycin) into cysts' cavities and argon coagulation of residual malformation tissues. EXIT procedure (ex-utero intrapartum treatment) was performed in one case - trachea was intubated within a delivery room under preserved placental blood circulation in a baby with prenatally diagnosed head and neck LM with upper airways compression. On the tenth day of life the tracheostomy was performed with following sclerotherapy. 
Clinical signs of VMs could be seen since the moment of birth in 18 out of 39 children $(46,2 \%)$ and AVMs in 3 out of $14(21,4 \%)$. The signs of VMs were skin lesions and superficial soft-tissue masses. AVMs presented with capillary spots. None of patients out of this group was diagnosed malformation during the first month of life, they all were referred to our clinic later with a provisional diagnosis of "hemangioma". As far as there were no functional disorders or complications in newborns with VMs and AVMs, treatment was not carried out in the neonatal period.

Cutaneous vascular lesions were seen in 53 (81.5\%) IHs patients during the first month of life, anyway, as were no signs of functional disorders the therapy was not assigned before 4 weeks old. Among 14 children with numerous skin lesions multifocal hepatic hemangiomas were diagnosed in 8 (57.1\%). Liver function was monitored, and regular sonography was performed to identify the hemangioma's activity level and its size. No correlation $(p=0.2)$ was found between the number of skin lesions and the severity of liver lesions (Fig. 2). Congestive cardiac failure suddenly occurs in one patient in the third week of life. The treatment of hemangioma was performed only with corticosteroids, beta-blocker therapy could not be started because the child required inotropic support.

Diffuse hepatic hemangiomas were diagnosed in 3 newborns. Two of them were manifested right after the birth with clinical signs of liver failure (elevated transaminases levels, hepatocellular jaundice, hepatomegaly, coagulopathy), hypothyreosis, and congestive cardiac failure. Sonography was used as a screening method for hepatic IH diagnosis. For additional visualization CT $(n=1)$ and MRI $(n=1)$ were performed, where total replacement of normal liver tissue by vascular neoplasms were seen (Fig. 3 ). Treatment of diffuse hepatic hemangiomas included corticosteroids, beta-blockers, replacement therapy for hypothyroidism, and urgent resuscitation.

9 newborns had clinical signs of $\mathrm{CHs}$. There were $\mathrm{CHs}$ of lower extremities $(n=2)$, liver $(n=3)$, upper extremities $(n=2)$, hairy part of the head $(n=2)$. CHs of superficial tissues were diagnosed on examination by typical diagnostic signs. Blood flow characteristics were determined by means of sonography; 2 patients required MRI for diagnosis. In 4 cases $(44.4 \%)$ CHs were diagnosed prenatally, others after birth $(n=5 ; 55.6 \%)$. Congenital liver hemangiomas were diagnosed prenatally as accidental findings in two cases and in early neonatal period in one case. To assess the blood flow and tumor size sonography was performed in dynamics, for clear diagnosis MRI was performed. All $\mathrm{CHs}$ had a tendency to spontaneous involution within the first month of life without reference to their location. Treatment was not carried out to newborns with focal hemangiomas as there were no functional liver disorders and the tumor involution was observed at ultrasound control examination.

KHEs were diagnosed in 7 newborns: tumors' locations were upper extremity $(n=1)$, shoulder and pectoral girdle $(n=3)$, neck and mediastinum $(n=1)$, neck, retroperitoneum and diaphragm $(n=1)$, liver $(n=1)$. The first clinical symptoms of the disease in 5 patients were visible since birth, in one case debut of the disease was at the age of 22 days. Tumor was visualized prenatally in 2 cases on routine ultrasound. KHE complications were thrombocytopenia (with platelet count of $8-10 \times 10^{9} / 1$ ) in all 7 newborns $(100 \%)$, pain syndrome, restriction of limb movement $(n=2)$, upper airway compression which required prolonged ( 2 weeks) intubation, $(n=1)$, hemothorax $(n=1)$ in a child with neck, retroperitoneum and diaphragm KHE, and congestive cardiac failure in patient with liver KHE. Repetitive platelet concentrates transfusions had temporary and short-term effect. Short courses of corticosteroids were more effective for thrombocytopenia correction. Surgical treatment was not possible for the reason of the infiltrative tumor's growth and large volume of different tissue involved. Patients underwent prolonged courses of betablockers and vincristine outside the newborn period.

It was established that the complications of vascular anomalies in newborn period correlates with the localization of lesions (Pearson's criterion 0.71, $\mathrm{p}<0.05$ ), with the size of mass (Pearson's criterion 0.53, $\mathrm{p}<0.05$ ), and with type of vascular malformation or tumor (Pearson's criterion is $0.64, \mathrm{p}<0.05$ ).

\section{DISCUSSION}

Congenital vascular malformations have diverse clinical manifestations, sometimes only as a vascular spot, in other cases with symptomatic growing with critical disfigurements and mass, that may cause life-threatening conditions [8]. Among 238 children with vascular anomalies that were included to our study clinical presentation at the age of 0 -28 days was seen in 170 (60.5\%), whereas only 14 (8.2\%) newborns required treatment. The indications for the treatment were clinically reasoned by development of dangerous complications, such as upper airways compression with respiratory failure when malformations were located within neck or head tissues; liver failure, hypothyreosis, cardiovascular failure in children with liver affection and thrombocytopenia in patients with aggressive vascular tumor - kaposiform hemangioendothelioma.

It is not only crucial to choose the optimal treatment method for vascular anomalies, but also to find an appropriate age for initiation of treatment. It is important to realize that not all vascular anomalies respond the treatment [9]. Moreover, they don't require treatment in all cases. Only the fact the child is diagnosed a vascular anomaly cannot become an indication for urgent surgical resection. Expectant management is a rational option in most cases, rather than impulsive treatment without comprehending the etiology and pathogenesis of the disease [10]. Some treatment methods that are used for vascular anomalies are aggressive, e.g. embolization may result into tissue ischemia; agents for sclerotherapy injected into vessels might cause tissues disruption, inflammation and encourage clotting. When large area is affected it may result into severe operational trauma. Most of LMs are isolated and don't threaten the life of a child. That is why 


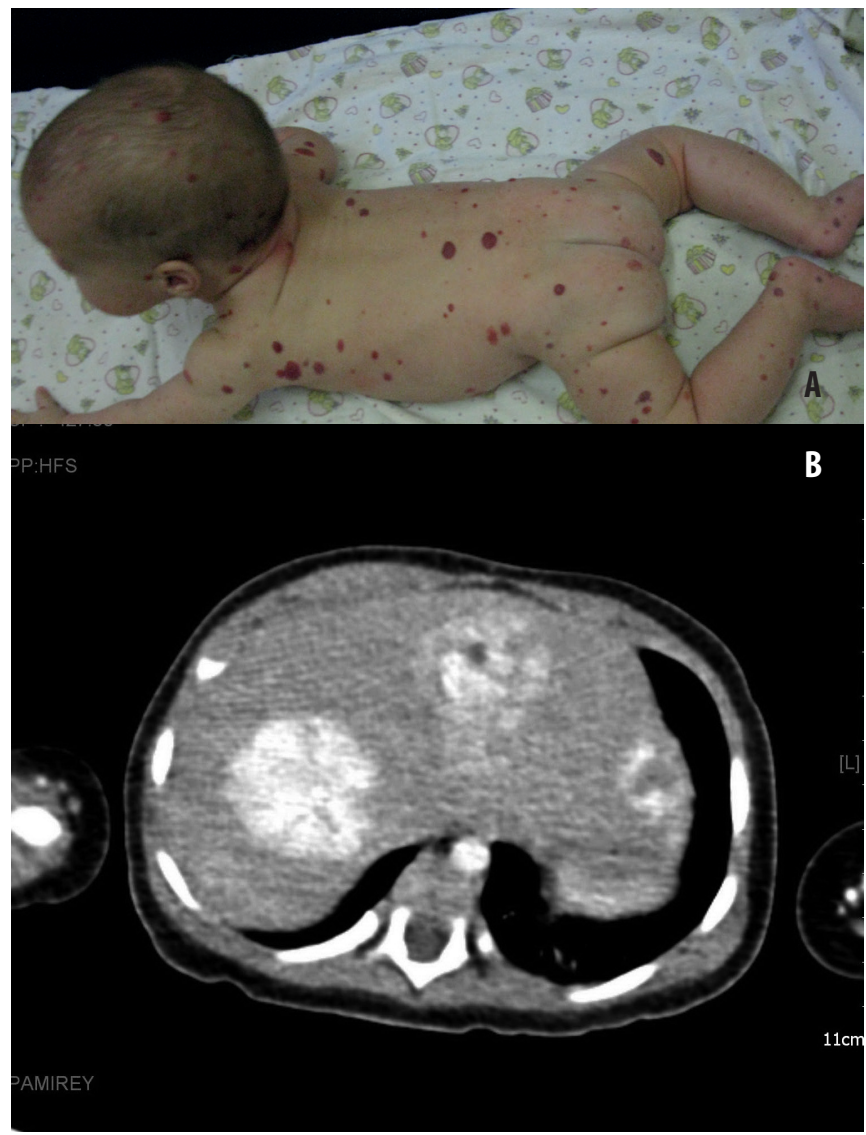

Fig. 2. Representative example of multiple infantile hemangioma. A Photo, multiple cutaneous lesions. B - axial CT, several hepatic lesions with intervening normal hepatic parenchyma.

it is reasonable to avoid surgical resections or sclerotherapy in children of young age as LMs are typically located near vital organs and anatomical structures and therefore surgical interventions can result into vital functions compromise. [11]. According to the literature data, in about $50 \%$ cases, LMs are visible at birth. These data correlate with the results of our study: $53.8 \%$ cases of head and neck
LMs were diagnosed in newborns, $6.4 \%$ of them required intubation and/or tracheostomy. Once a tracheotomy has been performed, it is common to wait until the airway is large enough to compensate for the initial cause of the obstruction This process may take many ears and it is common for children to need tracheostomy tube until the teenage years and sometimes ever longer [9]. For this reason, we performed partial resection and additional sclerotherapy in newborns in order to make decannulation procedure possible in early period. Recently, the frequent use of prenatal ultrasound has increased the likelihood of in utero diagnosis and, also, the use of ex utero intrapartum treatment procedures to secure the airway at birth in cases with upper airway obstruction [12].

VMs, that are sometimes called "cavernous hemangiomas" or "cavernomas", can present their first signs in early age, though the peak of clinical presentation appears by the age of 20 years. Less than $10 \%$ of extremities VMs are clearly seen before adolescence. According to this data in most cases there is no need in urgent therapy for newborns [13].

AVMs can manifest in newborns by a pink or red spot. They can in no way be distinguished from capillary spots. Rapid growth either complications development are not common for AVMs in young children therefore there is no need in urgent treatment [14].

Along first two months of life almost all IHs double their size. In a period of rapid growth, it can be seen how local IH covering $4 \mathrm{sm}^{2}$ turns into segmental IH covering $40 \mathrm{sm}^{2}$ [15]. IHs complications are related to their rapid growth in proliferation phase. Hepatic hemangiomas (infantile and congenital) are benign vascular tumors. The clinical behavior can be innocuous or life-threatening [16]. There is a mortality of $16 \%$ among children with multifocal and diffuse hepatic infantile hemangioma. Most of them had diffuse form, abdominal compartment syndrome, and heart failure. Being a potentially lethal conditions they require active treatment tactics in early age [17]. IH is high flow vascular tumors and can cause cardiac failure,



Fig. 3. Diffuse hepatic hemangioma. Coronal postcontrast MRI showing extensive vascular lesion replaces the hepatic parenchyma with little liver apparent. 
it will develop during the proliferation period as shunting increases. Acquired consumptive hypothyroidism is specific for hepatic infantile hemangioma via high expression of type 3 iodothyronine deiodinase that inactivates the thyroid hormone [16]. Consumptive hypothyroidism does not occur in congenital hemangioma (hepatic or any other location).

$\mathrm{CHs}$ are rare tumors; their true incidence is unknown as only isolated cases are described in the literature. Moreover, not all authors identify such nosological unit [18]. 9 patients with CHs were included into our study, which amounts $3.2 \%$ of all clinical cases. CHs appear at the twelfth week of gestation therefore are usually diagnosed by fetal sonography. They reach their maximum size within terminal weeks of pregnancy. After, involution of CHs is observed during baby's first month of life. Consequently, dynamic observation is required.

The most dangerous complications of KHE relate to coagulation disorders stemming from thrombocytopenia, which is known as Kasbah-Merritt syndrome (phenomenon) [19]. When tumor resection is possible the hematological disorders are resolved the same day. Though in most cases radical tumor excision is not possible for surrounding tissues infiltration and tumor size. Heparin is contraindicated that was proved by experimental studies. Platelet concentrate hasn't got therapeutic effect in newborns with hemangioendotheliomas as can provoke fast tumor growth, considerable platelets disruption, which half-life period after transfusion ranges from 1 to 24 hours. Drug treatment should be started as soon as possible. That will allow decreasing tumor size and reducing hematological complications to a minimum. [20]. Nevertheless, by contrast with treatment tactics of IH, there are no international standards for borderline tumors treatment. Positive impact of steroids in newborns for thrombocytopenia correction was observed, though the extensive further treatment was nevertheless required.

\section{CONCLUSIONS}

It is challenging to receive a diagnosis of vascular anomaly in newborns as specific signs are absent and there are limitations for additional methods of examination. Clinical signs of vascular anomalies in neonatal period were seen in $57.5 \%$ children with vascular anomalies, and only $2.9 \%$ of them required treatment. Urgent treatment is advantageous only in cases when life-threatening complications develop, such as airways obstruction in newborns with head and neck LMs, cardiac and/or liver failure in patients with defuse and multifocal hepatic hemangiomas, and severe thrombocytopenia in children with kaposiform hemangioendothelioma.

\section{REFERENCES}

1. Steele L., Zbeidy S., Thomson J. et al. How is the term haemangioma used in the literature? An evaluation against the revised ISSVA classification. Pediatr Dermatol. 2019; 18:1-6.

2. Lee B.B., Laredo J. Hemangioma and venous/vascular malformations are different as an apple and an orange! Acta Phlebologica. 2012; 13(1):1-3.
3. Mulliken J., Glowacki P. Hemangiomas and vascular malformations in infants and children. A classification based on endothelial characteristics. Plast Reconstr Surg 1982; 69:412-422.

4. Rudenko A.V., Vitovs'kyĭ R.M., Isaienko V.V. et al. The mitral valve abscess, which simulated the left ventricle tumor. Klin Khir. 2007; 9:58-60.

5. Bojchuk T.M., Yermolenko S.B., Fedonyuk L.Y.Y. et al. The magnitude of linear dichroism of biological tissues as a result of cancer changes. Proc.SPIE. Materials of the 10th International Conference Correlation Optics. 2011;83381.

6. Wiegand S., Eivazi B., Barth P.J. et al. Pathogenesis of lymphangiomas. Virchow's Arch. 2008; 453:1-8.

7. Perkins J.A. New frontiers in our understanding of lymphatic malformations of the head and neck: Natural history and basic research. Otolaryngol Clin North Am. 2018; 51:147-158.

8. SadickM., WohlgemuthW., Huelse R. etal. Interdisciplinary Management of Head and Neck Vascular Anomalies: Clinical Presentation, Diagnostic Findings and Minimalinvasive Therapies. European J of Radiol Open. 2017; 4:63-68.

9. Waner M.0. Multidisciplinary Approach to the Management of Lymphatic Malformations of the Head and Neck. Otolaryngol Clin North Am. 2018; 51(1):159-172.

10. Burrows P. Endovascular Treatment of Slow-FlowVascular Malformations. Techniques in Vascular and Interventional Radiology. 2013; 16(1):12-21.

11. Rickert S.M., Diallo A.M., Scheuermann-Poley C. et al. Lymphatic malformations of the airway. Otolaryngol Head Neck Surg. 2013; 149(1):156-60.

12. Ueno S., Fujino A., Morikawa Y. et al. Indications for tracheostomy in children with head and neck lymphatic malformation: analysis of a nationwide survey in Japan. Surg Today. 2019; 49(5):410-419.

13. Dasgupta R., Patel M. Venous malformations. Seminars in Pediatr Surg, 2014; 23(4):198-202.

14. Hawkins C.M., Chewning R.H. Diagnosis and Management of Extracranial Vascular Malformations in Children: Arteriovenous Malformations, Venous Malformations, and Lymphatic Malformations. Semin Roentgenol. 2019; 54(4):337-348.

15. Liang M., Frieden I. Infantile and congenital hemangiomas. Seminars in Pediatr Surg, 2014; 23(4):162-167.

16. lacobas I., Phung T.L., Adams D.M., Trenor C.C. et al. Guidance Document for Hepatic Hemangioma (Infantile and Congenital) Evaluation and Monitoring. J Pediatr. 2018; 12(203):294-300.

17. Rialon K., Murillo R., Fevurly R., Kulungowski A. et al. Impact of Screening for Hepatic Hemangiomas in Patients with Multiple Cutaneous Infantile Hemangiomas. Pediatric Dermatol. 2015; 32(6):808-812.

18. Boull C., Maguiness S.M. Congenital hemangiomas. Semin Cutan Med Surg. 2016; 35(3):124-7.

19. Schmid I., Klenk A.K., Sparber-Sauer M. et al. Kaposiform hemangioendothelioma in children: a benign vascular tumor with multiple treatment options. World J Pediatr. 2018; 14(4):322-329.

20. Tlougan B., Lee M., Drolet B. et al. Medical Management of Tumors Associated With Kasabach-Merritt Phenomenon. Journal of Pediatric Hematology: Oncology. 2013;35(8):618-622.

The work is carried out within the framework of the initiative research work of the Pediatric Surgery Department of Bogomolets National Medical University "Development and implementation of enhanced surgical management for young children with vascular malformations, that involves minimal invasive and combined treatment options with pathogenetic feasibility" (state registration No. 0113U004671). 


\section{ORCID and contributionship:}

Iryna M. Benzar: 0000-0001-7637-7769 A, D, F

Anatolii F. Levytskyi: 0000-0002-4440-2090 D, F

Daria S. Diehtiarova: 0000-0002-2356-0874 B,E,F

Larysa Ya. Fedoniuk: 0000-0003-4910-6888 C,E

Yaroslav S. Stravskyy: 0000-0001-6541-9097 C, F

Daria A. Merkulova: 0000-0002-5021-2349 E,F

Petro A. Sas: 0000-0003-1649-4087 ${ }^{\mathrm{C}}$

\section{Conflict of interest:}

The Authors declare no conflict of interest.

\section{CORRESPONDING AUTHOR}

\section{Iryna M. Benzar}

Bogomolets National Medical University

10G Vitryani Gory str, apt. 32, 04132 Kyiv, Ukraine

tel: +0380951295882

e-mail: ira_benzar@yahoo.com

Received: 22.04 .2020

Accepted: 07.07.2020

\footnotetext{
A - Work concept and design, B - Data collection and analysis, C - Responsibility for statistical analysis,
}

D -Writing the article, $\mathbf{E}$-Critical review, $\mathbf{F}$ - Final approval of the article 